\section{Behavioral effects of morphine and methadone in rhesus monkeys*}

\author{
GEORGE BIGELOW and TRAVIS THOMPSON \\ University of Minnesota, Minneapolis, Minn. 55455
}

Two rhesus monkeys, working on fixed-ratio schedules for appetitive reinforcement, were given injections of morphine sulfate and methadone hydrochloride. Morphine was the more potent in decreasing operant responding. Responding was restored sooner following methadone injections than following morphine injections. Response-decreasing potency does not correspond to analgesic potency.

The opiate and opioid drugs have a wide variety of psychopharmacological actions. They are analgesics (Lasagna, 1964; Jaffe, 1970); they serve as reinforcers to maintain operant behavior (Schuster \& Thompson, 1969); and they alter operant behavior maintained by both appetitive and aversive contingencies (McMillan, Wolf, \& Carchman, 1970; McMillan \& Morse, 1967; Verhave, Owen, \& Robbins, 1959; Hill et al, 1957).

Experimental attention has generally been attracted to the first two of these actions, due to the drugs' clinical use and dependence liability. Recently, however, methadone use has become widespread in treating opiate dependence, a problem involving a major behavioral consideration. One aim of such treatment is to allow the individual to function adequately within society (Einstein, 1970). Comparisons of the drugs' relative behavioral effects have, thus, become relevant. Relative potencies in the different aspects of psychopharmacological action need not be constant. These different aspects may be independent.

The present study compares the potencies of morphine and methadone in their effects upon operant behavior maintained by appetitive reinforcement in rhesus monkeys. Prior studies have indicated that these compounds will generally have a dose-dependent depressant effect upon such behavior (McMillan, Wolf, \& Carchman, 1970; McMillan \& Morse, 1967; Hill et al, 1957).

\section{EXPERIMENT 1}

Subject

One adult male rhesus monkey, weighing $10 \mathrm{~kg}$, and with extensive experience in this behavioral situation, served as the $S$.

\section{Apparatus}

The $S$ lived and was tested in a wire-mesh cubicle, $3 \mathrm{ft}$ on all sides.

* This research was supported by Grants MH-15349, MH-14112, and MH-08565 from the National Institute of Mental Health to the University of Minnesota.
One wall of the chamber served as the work panel, equipped with three pull-type (Lindsley) manipulanda, each with an associated stimulus light directly above. Each manipulandum corresponded to a different reinforcer a food biscuit (Purina Monkey Chow), a drink of water $(1.5 \pm 0.5 \mathrm{cc}$ delivered through a drinking tube), or a dried apricot.

An artificial screen wall on one side of the chamber served as a squeeze panel by which the $S$ could be pulled to the side for injections.

Other monkeys were in adjacent chambers, permitting social contact through the screening. Solid-state control and recording equipment was located in an adjoining room.

\section{Procedure}

The $S$ received all nutritional intake during the experimental sessions, except for occasional vitamin and fresh fruit supplements. Sessions occurred twice daily, 7 days per week, at 12 -h intervals, and terminated with the first reinforcement after $2 \mathrm{~h}$ had elapsed.

A fixed number of responses was required for each reinforcement (a fixed-ratio schedule). The fixed ratio (FR) values were: FR 75 for food, FR 20 for water, and FR 50 for apricots. These reinforcers were available on a nonreversible option basis (Findley, 1962). At the start of a session, or immediately following a reinforcement, all three stimulus lights were illuminated (i.e., responses on all three levers were effective). As soon as the $S$ responded on one manipulandum, responses on the other two had no programmed consequence, and their lights were extinguished until the FR was completed for the selected reinforcer and reinforcement had occurred.

Dose-response relations. Three doses each of morphine sulfate and methadone hydrochloride were examined: $0.125,0.250$, and $0.500 \mathrm{mg} / \mathrm{kg}$. Volumes were $0.50 \mathrm{cc}$. Injections were given intramuscularly, 5 min before the start of the first of the two daily sessions. The doses were given in a mixed order. At least three noninjection days intervened between drug injections. Saline was administered twice during the drug series. This drug series was dicontinued after one trial of each dosage.

Chronic administration. For $\mathbf{3 6}$ days, $0.50 \mathrm{mg} / \mathrm{kg}$ of either morphine or methadone was given daily by the same procedure described above. Each drug was administered for 3 consecutive days, then the other drug was administered for 3 consecutive days. This cycle was repeated six times.

\section{Results}

Dose-response relations. The total responses per session for each injection day, as well as the median interquartile range and range for 24 noninjection control days are shown in Fig. 1. The data for the two daily sessions are presented separately. Morphine consistently produces a greater depression of responding than methadone. The chance of this happening in all six comparisons by chance (i.e., if the two drugs were actually of equal behavioral potency) is less than 0.02

In the first of the two daily sessions there is, for both drugs, an inverse relation between responding and dosage. This graded dose relationship still holds for morphine in the second session (more than $12 \mathrm{~h}$ after the drug administration). However, after methadone, in this second session responding has returned to control levels. After the $0.125-\mathrm{mg} / \mathrm{kg}$ dose of methadone, responding is above the range for the 24 noninjection control days.

Chronic administration. Figure 2 shows the total responses in each session during the chronic drug administration phase. During the first

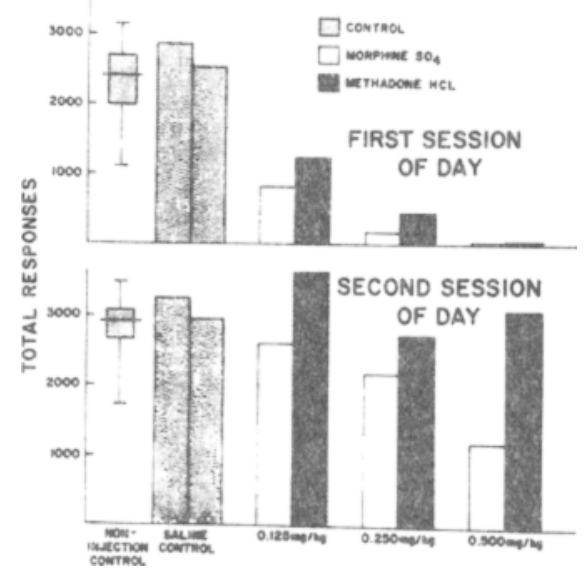

Fig. 1. Responding during the two daily sessions on control days and following various doses of morphine and methadone. 


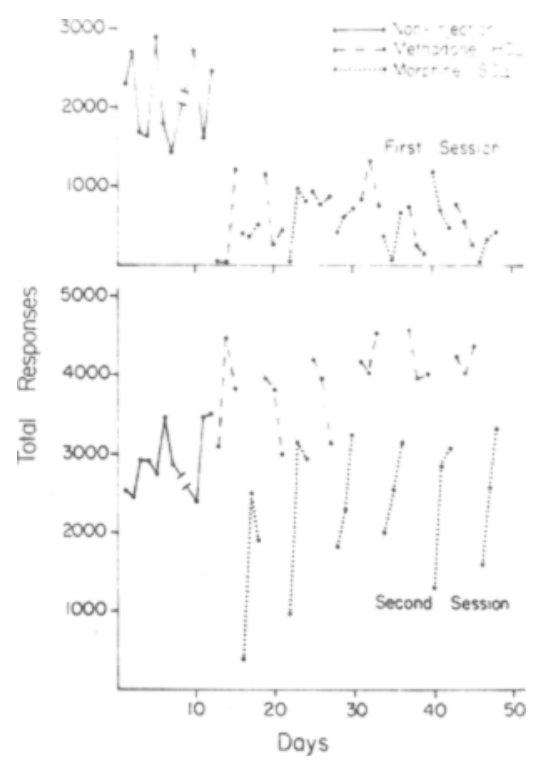

Fig. 2. Responding during the two daily sessions for the chronic drug administration phase.

daily session, both drugs produce a profound reduction in responding (no overlap with control sessions), and their effects are not significantly different $(t=1.04)$.

In the second session, morphine still reduces responding significantly below control levels $(\mathrm{p}<.02, t=2.67$, two-tailed); methadone no longer does so. In fact, responding after methadone is significantly above that on control days during the second session $(p<.001, t=5.94$, two-tailed). The difference in responding after morphine as compared to methadone is, of course, also significant at the .001 level $(t=7.40$, two-tailed $)$.

EXPERIMENT 2

The initial study suggested that an important aspect of the difference between morphine's and methadone's behavioral potencies lay in the duration of their effect. This second experiment sought to examine the time course of the return of responding which had been depressed by the drugs, and also to extend the study to a second animal.

\section{Subject}

One adult male rhesus monkey, weighing $10 \mathrm{~kg}$, served as the $S$.

\section{Apparatus}

The $S$ was housed in an experimental space similar to that described in Experiment 1, except that the work panel had only one manipulandum and stimulus light. Below this there was a hopper for delivery of $1-\mathrm{g}$ Noyes food pellets.

Procedure

The $S$ received all of his food during the experimental sessions, except for occasional fresh fruit supplements. Water was freely available. Half-hour sessions, during which a fixed-ratio schedule for food reinforcement (FR 30) was in effect, occurred repeatedly throughout the day with a $11 / 2-h$ intersession period.

The behavioral effects of morphine sulfate and methadone hydrochloride were compared at a dosage of $0.50 \mathrm{mg} / \mathrm{kg}$. Each drug was administered five times, in a mixed order, via intramuscular injection in a 0.5 -cc volume.

\section{RESULTS}

Both drugs produced a suppression of responding. Their potencies in doing so were compared by comparing the number of sessions which had elapsed before substantial responding again occurred. Figure 3 shows the mean number of responses occurring in each of the first four daily sessions following morphine or methadone injections. This covers a period of $61 / 2 \mathrm{~h}$ following drug administration.

The response suppression produced by methadone dissipates more rapidly than that for morphine. Substantial responding occurs in the third session after methadone, but not until the fourth session after morphine. The response rates in the third session are significantly different, as indicated by a $t$ test $(p<.02$, two-tailed $)$.

\section{DISCUSSION}

Both experiments indicate that the behavioral potencies of morphine and methadone differ. Both decrease response rate, but morphine is consistently the more potent. The greater response reduction produced by morphine seems to be related to its longer duration of behavioral action. Responding is restored several hours earlier following methadone than following morphine.

McMillan, Wolf, \& Carchman (1970) reported a comparison of morphine and methadone on schedule-controlled behavior in the pigeon. They described the two drugs as being equipotent (their data, however, show methadone to be more potent). The discrepancy between their results with pigeons and the present findings with monkeys might be due to their relatively short experimental sessions. The present study suggests that the duration of behavioral effect is an important difference between the two compounds.

Clinical reports concerning methadone treatment of opiate dependence suggest also that methadone is not very potent in altering operant behavior. Dole (1970) has indicated that human methadone patients show "normal" performance on neuromotor coordination, reaction

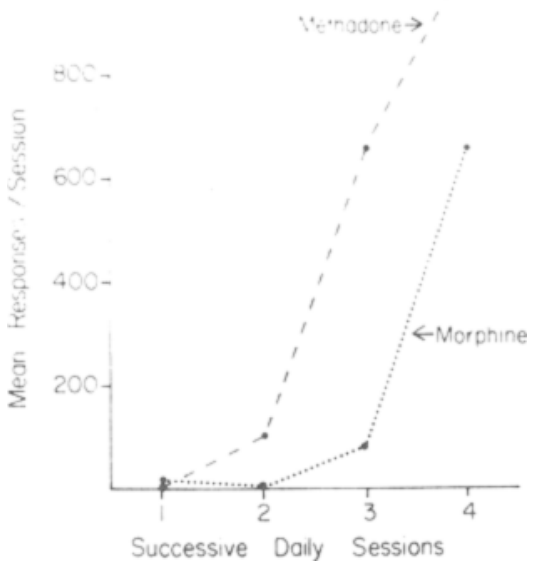

Fig. 3. Average responses in the first four sessions following injections of morphine and methadone.

time, and vigilance tasks. The relative weakness of methadone in decreasing operant responding would certainly be a valuable attribute insofar as its clinical use in treating opiate dependence is concerned.

The present results emphsaize that a drug may exert several independent actions. Though the two drugs are equipotent for analgesic purposes (Lasagna, 1964; Jaffe, 1970), they differ in their potencies in decreasing operant behavior. The two drugs also appear to differ in reinforcing potency. Jaffe (1970) has pointed out that methadone is less preferred by illicit users. Collins \& Weeks (1965) have prepared rats with intravenous catheters and allowed them to self-administer morphine and methadone. Methadone was self-administered less frequently. It is unclear whether any relation exists between response-decreasing potency and reinforcing potency. To answer this question, it would be valuable to examine a wider range of opiate and opioid compounds.

\section{REFERENCES}

COLLINS, R. J., \& WEEKS, J. R. Relative potency of codeine, methadone and dihydromorphinone to morphine in self-maintained addict rats. Archiv für Pharmakologie und Experimentelle Pathologie, 1965, 249, 509-514.

DOLE, V. P. Research on methadone maintenance treatment. International Journal of the Addictions, 1970. 5 . 359-369.

EINSTEIN, S. (Ed.) Second Nationa Methadone Maintenance Conference. International Journal of the Addictions, $1970,5,341-591$.

FINDLEY, J. D. An experimental outline for building and exploring multi-operant behavior repertoires. Journal of the Experimental Analysis of Behavior, 1962, 5, 113-166.

HILL, H., PESCOR, F.. BELLEVILLE, R. \& WIKLER, A. Use of differential 
bar-pressing rates of rats for screening analgesic drugs. I. Techniques and effects of morphine. Journal of Pharmacology \& Experimental Therapeutics, 1957, 120. 388-397.

JAFFE $J$ Narcotic analgesics. In $L$. Goodman and A. Gilman (Eds.). The pharmacological basis of therapeutics. New York: Macmillan, 1970. Pp. 237.275.

LASAGNA, L. The clinical evaluation of morphine and its substitutes as analgesics.
Pharmacology Review, 1964, 16, 47-83. McMILLAN, D.. \& MORSE, W. Some effects of morphine and morphine antagonists on schedule-controlled behavior. Journal of Pharmacology \& Experimental Therapeutics, 1967, 157, 175-184.

MCMILLAN, D., WOLF, P., \& CARCHMAN, $R$. Antagonism of the behavioral effects of morphine and methadone by narcotic antagonists in the pigeon. Journal of Pharmacology \& Experimental Therapeutics, 1970, 175 , 443.458

SCHUSTER, C. R., \& THOMPSON, T. Self-administration of and behavioral dependence on drugs. Annual Review of Pharmacology, 1969, 9, 483-502.

VERHAVE, T., OWEN, J., \& ROBBINS, E. The effect of morphine sulfate on avoidance and escape behavior. Journal of Pharmacology \& Experimental Therapeutics, 1959, 125, 248-257.

\section{CURRENT LITERATURE OF DRUG EFFECTS ON BEHAVIOR}

BRACKBILL, R. M. (Franklin and Marshall College, Lancaster, $\mathrm{Pa}$. 17604), \& BROOKSHIRE, K. H. Conditioned taste aversions as a function of the number of CS-US pairs. Psychonomic Science, 1971, 22, 25-26.

CAPALDI, E. J. (Purdue University, L afayette, Ind. 47907), \& SPARLING, D. L. Amobarbital and the partial reinforcement effect in rats: Isolating frustrative control over instrumental responding. Journal of Comparative and Physiological Psychology, 1971, 74, 467-477.

CROW, L. T. (Western Washington State College, Bellingham, Wash. 98225). Alcohol ingestion in rats following median eminence lesions. Psychonomic Science, 1971, 22, 36-37.

DUNCAN, P. M. (Department of Physiology and Biophysics, University of Washington, Seattle, Wash. 98105). Effect of temporary septal dysfunction on conditioning and performance of fear responses in rats. Journal of Comparative and Physiological Psychology, 1971, 74, 340-348.

ELIAS, M. F. (Center for the Study of Aging and Human Development, Duke University Medical Center, Durham, N.C. 27706 ), BLENKARN, G. D., SIMMERMAN, S. J., \& MARSH, G. R. Administration of inhalation anesthetics to small animals: Some problems and solutions. Behavior
Research Methods \& Instrumentation, 1971, 3, 70-71.

FRUMKIN, K. (McGill University, Montreal 110, Quebec, Canada). Interaction of $\mathrm{LiCl}$ aversion and sodium-specific hunger in the adrenalectomized rat. Journal of Comparative and Physiological Psychology, 1971, 75, 32-40.

GALLUSCIO, E. H. (554 Gloria Drive, Baton Rouge, La. 70815 ). Retrograde amnesia induced by electroconvulsive shock and carbon dioxide anesthesia in rats: An attempt to stimulate recovery. Journal of Comparative and Physiological Psychology, 1971, 75, 136-140.

GERBEN, M. J. (U.S. Army Research Institute of Environmental Medicine, Natick, Mass. 01760), JONES, L. G., \& SMOAKE, J. A. Behavioral tolerance of squirrel monkeys to hypoxia: A model for evaluating drug therapy. Behavior Research Methods \& Instrumentation, 1971, 3, 10-12.

KESNER, R. P. (University of Utah, Salt Lake City, Utah 84112). ECS as a punishing stimulus: Dependency on retrograde amnesia, duration of anterograde amnesia, and intensity of pain. Journal of Comparative and Physiological Psychology, 1971, 74, 398-406.

KETTLEWELL, N. M. (University of Montana, Missoula, Mont. 59801), \& PAPSDORF, J. D. A role for cutaneous afferents in classical conditioning in rabbits. Journal of
Comparative and Physiological Psychology, 1971, 75, 239-247.

KHAVARI, K. A. (University of Wisconsin-Milwaukee, Milwaukee, Wis. 53201). Adrenergic-cholinergic involvement in modulation of learned behavior. Journal of Comparative and Physiological Psychology, 1971, 74, 284-291.

POTTS, W. J. (G. D. Searle \& Co., Chicago, Ill. 60680), \& EAST, P. F. A simple epidural cannula for the rat. Behavior Research Methods \& Instrumentation, 1971, 3, 136-137.

TRAFTON, C. L. (University of Arizona, Tucson, Ariz. 85721), \& MARQUES, P. R. Effects of septal area and cingulate cortex lesions on opiate addiction behavior in rats. Journal of Comparative and Physiological Psychology, 1971, 75, 277-285.

WRIGHT, W. E. (George Peabody College, Nashville, Tenn, 37203), \& FOSHEE, D. P., \& McCLEARY, G. E. Comparison of taste aversion with various delays and Cyclophosphamide dose levels. Psychonomic Science, 1971, 22, 55-56.

ZIFF, D. R., \& CAPALDI, E. J. (Purdue University, Lafayette, Ind. 47907). Amytal and the small trial partial reinforcement effect: Stimulus properties of early trial nonrewards. Journal of Experimental Psychology, 1971, 87, 263-269. 